\title{
Treatment of a patient with total urinary calculi: a case report
}

\author{
Jiajia Ma", Xuebao Zhang", Jipeng Wang, Zhongbao Zhou, Chunhua Lin \\ Department of Urology, The Affiliated Yantai Yuhuangding Hospital of Qingdao University, Yantai 264000, China \\ \#These authors contributed equally to this work. \\ Correspondence to: Chunhua Lin. Department of Urology, The Affiliated Yantai Yuhuangding Hospital of Qingdao University, No. 20 East \\ Yuhuangding Road, Yantai 264000, China. Email: linchunhua1980@163.com.
}

\begin{abstract}
Urinary tract stones are a general term for stone disease in various parts of the urinary system and are common diseases of the urinary system. According to the location of the stone, it is divided into kidney stones, ureteral stones, bladder stones, urethra stones. Total urinary calculi occur less frequently, usually accompanied by severe clinical symptoms and difficult to treat. A 76-year-old man was admitted to hospital with repeated bladder stones for 7 years, repeated dysuria with frequent urination and dysuria for 2 months. After admission, the patient improved the relevant examination, and the patient was finally diagnosed with total urinary calculi combined with infection. After a series of orderly and effective treatments, especially the completion of the one-stage operation, the patient recovered smoothly and was discharged. Complicated whole-course urolithiasis is relatively rare and a single operation of total urinary calculi is feasible.
\end{abstract}

Keywords: Total urinary calculi; infection; minimally invasive technique; case report

Submitted Oct 29, 2019. Accepted for publication Nov 21, 2019.

doi: $10.21037 /$ tau.2019.11.23

View this article at: http://dx.doi.org/10.21037/tau.2019.11.23

\section{Introduction}

Urinary tract stones are a general term for stone disease in various parts of the urinary system and are common diseases of the urinary system (1). According to the location of the stone, it is divided into kidney stones, ureteral stones, bladder stones, urethra stones. The formation of this disease is closely related to environmental factors, systemic diseases and urinary system diseases (2). Typical clinical manifestations include lumbar and abdominal cramps, hematuria, or symptoms of urinary tract obstruction and infection with frequent urination, urgency, and dysuria. Total urinary calculi occur less frequently, usually accompanied by severe clinical symptoms and difficult to treat. In such cases, a surgical approach is usually used as curative treatment (3). We reported a case of total urinary calculi that passed through a series of progressive treatments and rehabilitation in our hospital. We present the following case in accordance with the CARE guideline (4).

\section{Case presentation}

A 76-year-old Chinese man with recurrent bladder stones for 7 years, recurrent dysuria with frequent urination and dysuria for 2 months as the chief complaint was admitted to our hospital. For many years, patients have been suffering from this disease, and have been hospitalized many times to relieve symptoms. The patient was unable to endure the same symptoms 7 years ago and went to the hospital for "cystolithotomy". The above symptoms reappeared 4 years ago. He was again treated with "cystolithotomy". One year ago, because the symptoms continued to worsen and did not relieve and emergency hospitalization, the diagnosis was "bladder stones and vesical fistula", and he underwent "cystolithotomy + transurethral prostatectomy + bilateral double-J tube (D-J tube) implantation" treatment, it is worth mentioning that the patient has not replaced the D-J tube. In addition, the patient has a complicated past history and has been hospitalized for multiple diseases. These operations are mainly: inguinal hernia surgery, urethral rupture repair, intracranial aneurysm clipping. His family history is not remarkable.

The patient underwent abdominal computed tomography (CT) after admission (Figure 1). The results showed that: (I) bilateral kidney stones; (II) ureter D-J tube 


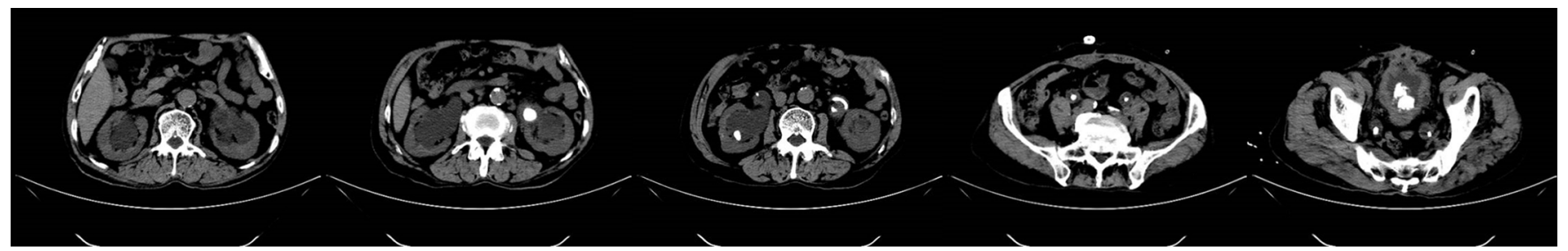

Figure 1 Abdominal computed tomographic scan of the patient.

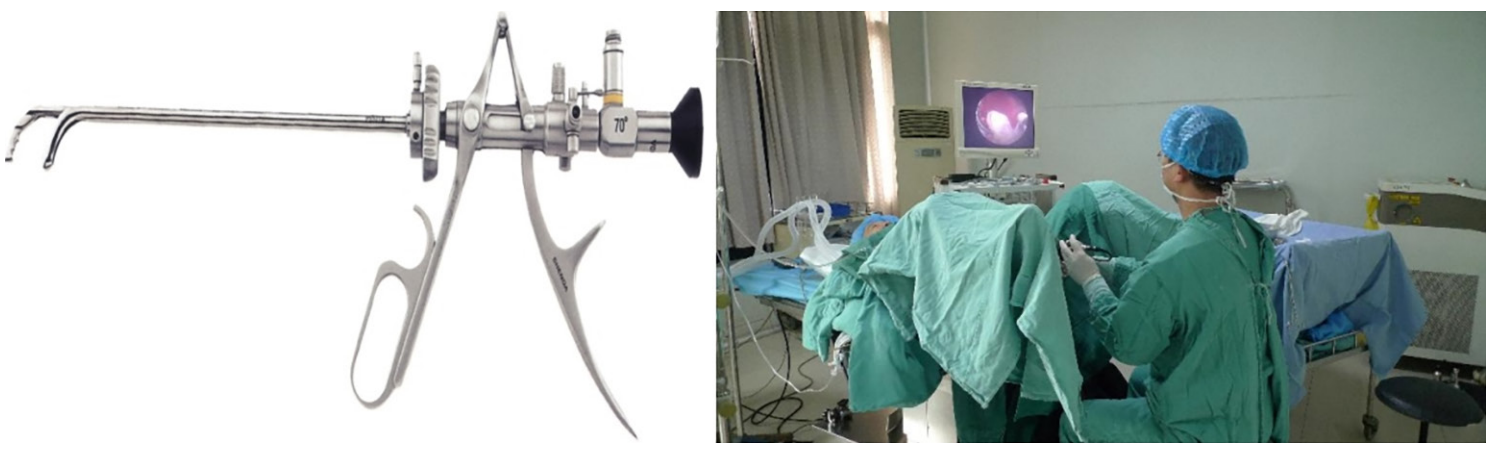

Figure 2 The cystoscope lithotripsy for bladder stones.

stones attachment; (III) bladder stones. Routine urianlysis shows: white blood cell (WBC) count $4,578 / \mu \mathrm{L}$; blood biochemistry: creatinine $(\mathrm{Cr}) 140 \mathrm{mmol} / \mathrm{L}$. The main positive sign of the patient's admission physical examination was pain in the left kidney area. According to the results of the series of examinations and past history, the patient is currently considering the total urinary calculi with severe infection, accompanied by renal dysfunction. In addition, depending on the patient's condition, the patient should rule out the possibility of the tumor, and we initially ruled out this possibility. Then how to treat patients in the next step is a question worth considering.

We first carried out some basic and temporary measures to improve the patient's symptoms. Our first treatment was to give catheterization and bilateral nephrostomy, which both led to grayish pyuria. At the same time, piperacillin tazobactam was actively used for anti-inflammatory treatment. After 1 week, the urine routine was reviewed: WBC $311.00 / \mu \mathrm{L}$, red blood cell (RBC) count $628.00 / \mu \mathrm{L}$, WBC 56/high power field (HPF), RBC 113/HPF, WBC+++, blood (BLD)+++.

After 3 days, the urine routine was reviewed again: WBC

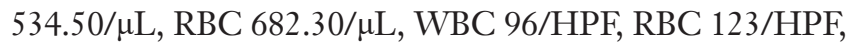

WBC+++, BLD+++. Review of serum Cr: $112 \mathrm{mmol} / \mathrm{L}$. In addition, bilateral nephrolithotomy drainage of about $3,600 \mathrm{~mL}$ of urine. As of our treatment, the patient's condition does not seem to improve significantly.

Faced with such a complicated condition, how to treat the patient in the next step becomes a problem that plagues us. Our trouble is whether to continue anti-infective treatment or surgical treatment. If surgery is selected, is it a staged operation or a one-stage operation, what is the order of stone treatment? Consider the patient's advanced age and general poor physical condition, the staging operation requires multiple operations with a long-time span, and patients may not be able to withstand multiple anesthesia and surgical blows. After our unanimous discussion, we fully decided to perform a surgical treatment after fully explaining our treatment plan to the patient's family and obtaining the consent of the patient's family. The patient underwent adequate preoperative preparation.

Bladder stones were first treated with cystoscope lithotripsy (Figure 2), and then ureteral D-J tube stones were treated with bilateral ureteroscope holmium laser lithotripsy (Figure 3). Finally, regarding the treatment of kidney stones, we considered that the kidney stones on the 

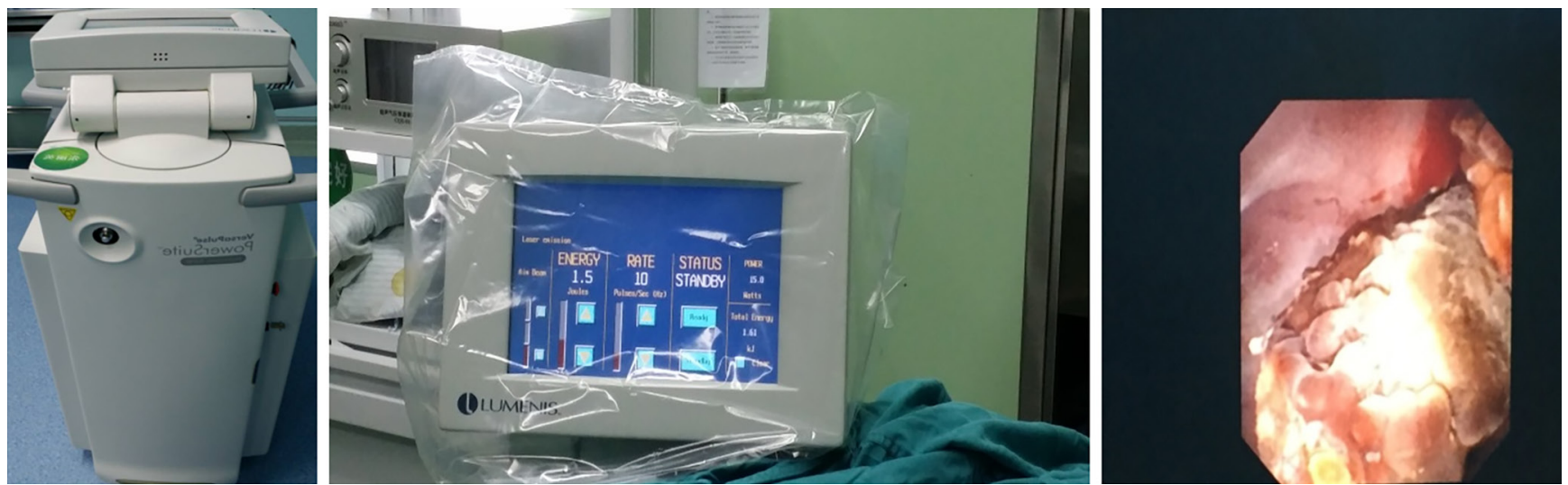

Figure 3 Bilateral ureteroscope holmium laser lithotripsy for ureteral D-J tube stones. D-J tube, double-J tube.
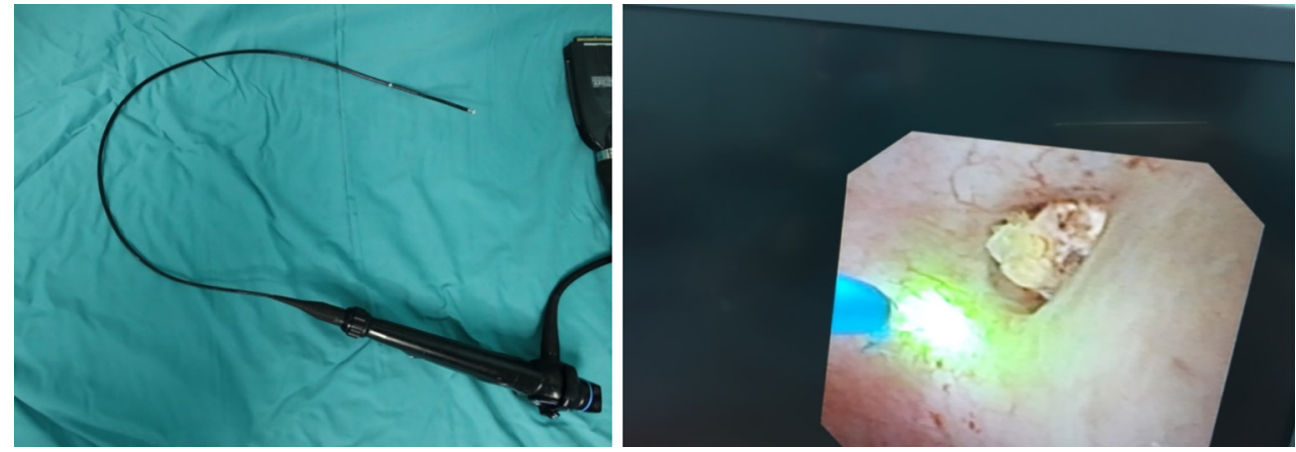

Figure 4 Treatment of right kidney stone with holmium laser lithotripsy with flexible ureteroscope.
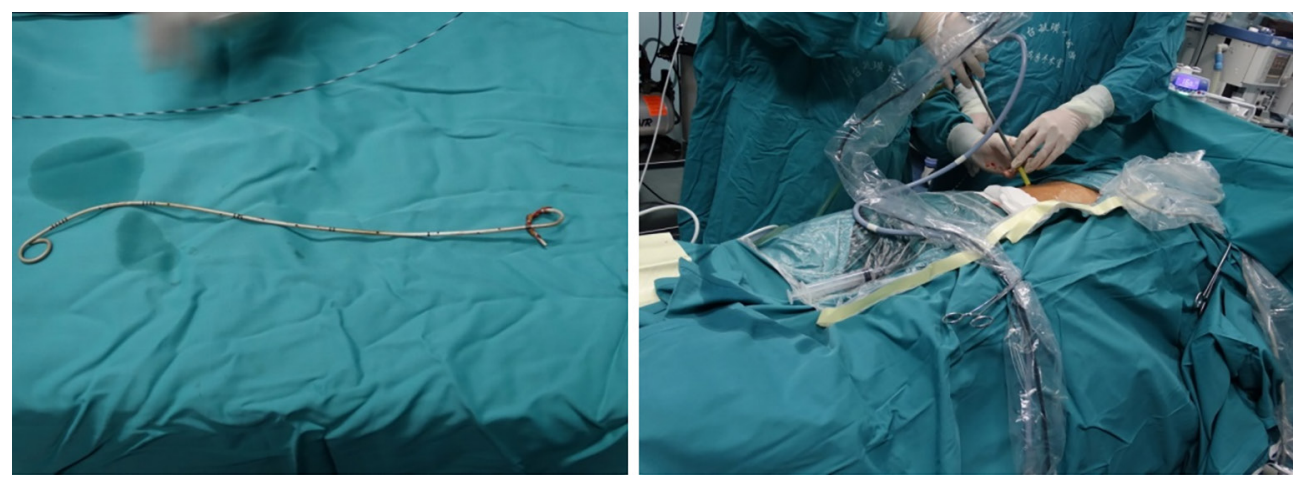

Figure 5 Percutaneous nephrolithotomy for left kidney stones.

right side were small, so we used the right ureteroscopic holmium laser lithotripsy to treat the right side of the stone (Figure 4). Considering that the kidney stones on the left side were large, we decided to adopt the left percutaneous nephrolithotomy (PCNL) (Figure 5). The entire procedure lasted approximately $5 \mathrm{~h}$ and the patient returned to the ward smoothly.

The patient's postoperative temperature was normal, the kidney function improved, and the symptoms disappeared. The plain film of kidney-ureter-bladder (KUB) on the 1st 
day after surgery showed no obvious stone residue, and the position of the bilateral D-J tube was acceptable (Figure 6). Moreover, we required the patient to go to the ground and resume normal eating on the 2 nd day after the operation. The patient recovered well, and the renal drainage tube was removed on the 7 th day after the operation and discharged successfully. In addition, it is worth mentioning that the composition of postoperative stones was magnum ammonium phosphate (Figure 7). The 12-month followup included ultrasonography examinations at 1, 2, 3, 6, 9 and 12 months postoperatively and showed that no stones

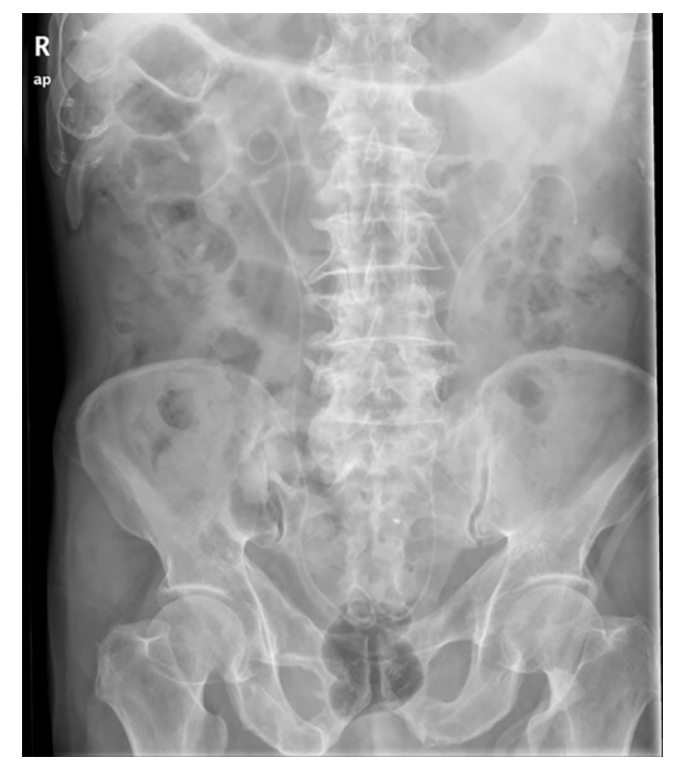

Figure 6 The postoperative KUB. KUB, kidney-ureter-bladder. formed, and no recurrence of previous symptoms.

\section{Discussion}

Patients with urinary calculi in the whole course are rare, and long stay in D-J tube and chronic lower urinary tract obstruction are the two main causes of stone formation (5-7). The patient-described in this article is a typical case. D-J tubes are widely used in urology. Long-term retention in the body can form a large number of stones with the D-J tube as the core. The cause of the formation of D-J tube attached stones is not clear, and may be related to the D-J tube material itself and its surface characteristics, urine characteristics, in vivo retention time and urinary tract infection (8-10). el-Faqih et al. analyzed the D-J tube taken from a large number of patients and found that the formation rate of adherent stones was $9.2 \%$ in the indwelling time less than 6 weeks, $47.5 \%$ in the $6-12$ weeks, and $76.3 \%$ in the 12 weeks (11). Urinary calculi caused by D-J tube retention are more common in both ends of the D-J tube and in the lumen. This kind of stone treatment is complicated, so it is necessary to prevent complicated whole-course urinary stones caused by D-J tube retention. Most scholars believe that the D-J tube indwelling course should be 2-6 weeks; there are also clinical studies to confirm that the D-J tube indwelling less than 14 days after the URL can not only reduce the uncomfortable reaction, but also reduce the amount of antibiotics used (12); Drink more water, acidify urine, and timely anti-infective treatment (13). Therefore, it is especially important to inform patients to change the D-J tube regularly and drink more water.

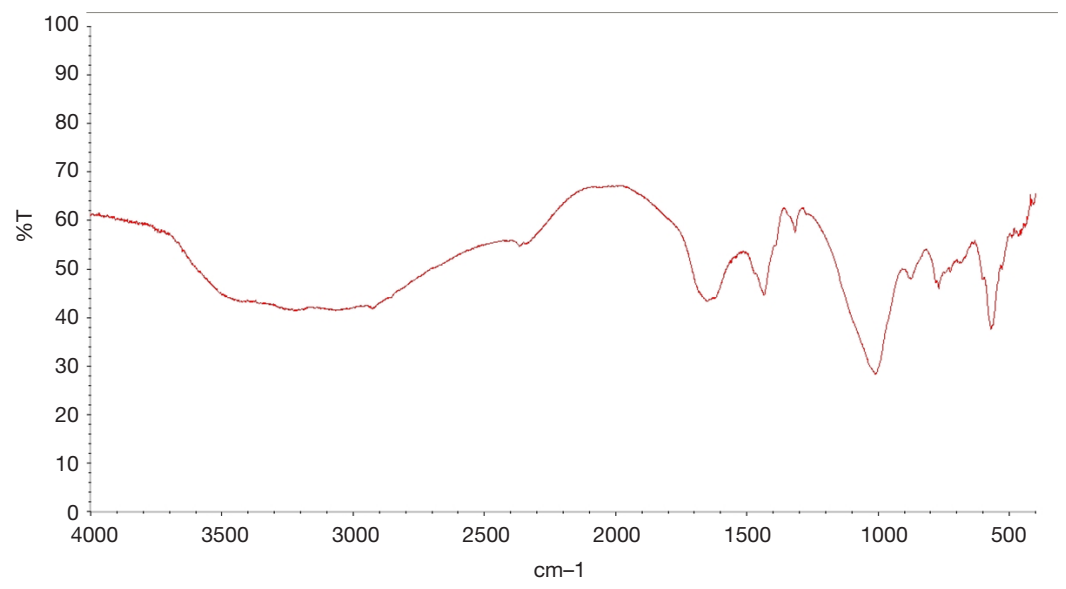

Figure 7 Stone composition analysis. 
In addition, the adequate drainage of various ostomy tubes before surgery and the application of preoperative antibiotics have effectively improved the patient's infection, which is an important condition for the success of the first operation $(14,15)$. Another important condition for successful surgery is the control of intraoperative low pressure (16). The main mechanism is that intraoperative low pressure can prevent excessive renal perfusion pressure, avoiding the perfusion solution containing bacteria and endotoxin absorbed by the renal regurgitation, causing postoperative fever and sepsis (17). Therefore, continuous and effective maintenance of intraoperative low pressure can reduce the occurrence of renal regurgitation and improve the safety of minimally invasive surgery for kidney stones.

Urinary tract stones caused by chronic lower urinary tract obstruction are more common, the most common is benign prostate hyperplasia $(\mathrm{BPH})$ with bladder stones, but the urinary calculi in the kidney, ureter, and bladder are rare. The treatment of such patients, considering the patient's surgical tolerance, long-term surgery and long-term pump lavage may bring serious complications, clinically generally choose staged surgery. However, the treatment of this group of patients also confirmed that a single operation of total urinary calculi is feasible. However, it should be noted here that when faced with a patient with a urinary calculi, our treatment is not a guideline, but is used as a case for reference. Because the patient's age, physical condition, operative time and patient's tolerance should be taken into account during the treatment, the specific treatment plan should also be based on this.

\section{Conclusions}

Complicated whole-course urolithiasis is relatively rare. With the maturity of minimally invasive treatment methods such as extracorporeal shock wave lithotripsy (ESWL), ultrasonic lithotripsy (URL) (holmium laser, shock wave, ultrasound, etc.) and PCNL, minimally invasive treatment of complex whole-course urolithiasis has become an ideal choice and has broad application prospects.

\section{Acknowledgments}

None.

\section{Footnote}

Conflicts of Interest: The authors have no conflicts of interest to declare.

Ethical Statement: The authors are accountable for all aspects of the work in ensuring that questions related to the accuracy or integrity of any part of the work are appropriately investigated and resolved. Written informed consent was obtained from the patient for publication of this case report and any accompanying images.

\section{References}

1. Schade GR, Faerber GJ. Urinary tract stones. Prim Care 2010;37:565-81, ix.

2. Trinchieri A. Urinary calculi and infection. Urologia 2014;81:93-8.

3. De Coninck V, Antonelli J, Chew B, et al. Medical expulsive therapy for urinary stones: future trends and knowledge gaps. Eur Urol 2019;76:658-66.

4. Riley DS, Barber MS, Kienle GS, et al. CARE guidelines for case reports: explanation and elaboration document. J Clin Epidemiol 2017;89:218-35.

5. Murthy KV, Reddy SJ, Prasad DV. Endourological management of forgotten encrusted ureteral stents. Int Braz J Urol 2010;36:420-9.

6. Dyer RB, Chen MY, Zagoria RJ, et al. Complications of ureteral stent placement. Radiographics 2002;22:1005-22.

7. Javali T, Nayak KA, Babu SMLP. Simultaneous antegrade and retrograde endoscopic surgery for benign prostatic hyperplasia with vesical calculi - a single-centre experience. Arab J Urol 2018;16:417-21.

8. Farsi HM, Mosli HA, Al-Zemaity MF, et al. Bacteriuria and colonization of double-pigtail ureteral stents: long-term experience with 237 patients. J Endourol 1995;9:469-72.

9. Reid G, Denstedt JD, Kang YS, et al. Microbial adhesion and biofilm formation on ureteral stents in vitro and in vivo. J Urol 1992;148:1592-4.

10. John T, Rajpurkar A, Smith G, et al. Antibiotic pretreatment of hydrogel ureteral stent. J Endourol 2007;21:1211-6.

11. el-Faqih SR, Shamsuddin AB, Chakrabarti A, et al. Polyurethane internal ureteral stents in treatment of stone patients: morbidity related to indwelling times. J Urol 1991;146:1487-91.

12. Getliffe KA, Mulhall AB. The encrustation of indwelling catheters. Br J Urol 1991;67:337-41.

13. Kunin CM, Chin QF, Chambers S. Formation of encrustations on indwelling urinary catheters in the elderly: 
a comparison of different types of catheter materials in "blockers" and "nonblockers". J Urol 1987;138:899-902.

14. Yoon GH, Bellman GC. Tubeless percutaneous nephrolithotomy: a new standard in percutaneous renal surgery. J Endourol 2008;22:1865-7; discussion 1869.

15. Feng MI, Tamaddon K, Mikhail A, et al. Prospective randomized study of various techniques of percutaneous

Cite this article as: Ma J, Zhang X, Wang J, Zhou Z, Lin C. Treatment of a patient with total urinary calculi: a case report. Transl Androl Urol 2019;8(6):764-769. doi: 10.21037/ tau.2019.11.23 nephrolithotomy. Urology 2001;58:345-50.

16. Alsmadi J, Fan J, Zhu W, et al. The influence of super-mini percutaneous nephrolithotomy on renal pelvic pressure in vivo. J Endourol 2018;32:819-23.

17. Zhong W, Leto G, Wang L, et al. Systemic inflammatory response syndrome after flexible ureteroscopic lithotripsy: a study of risk factors. J Endourol 2015;29:25-8. 